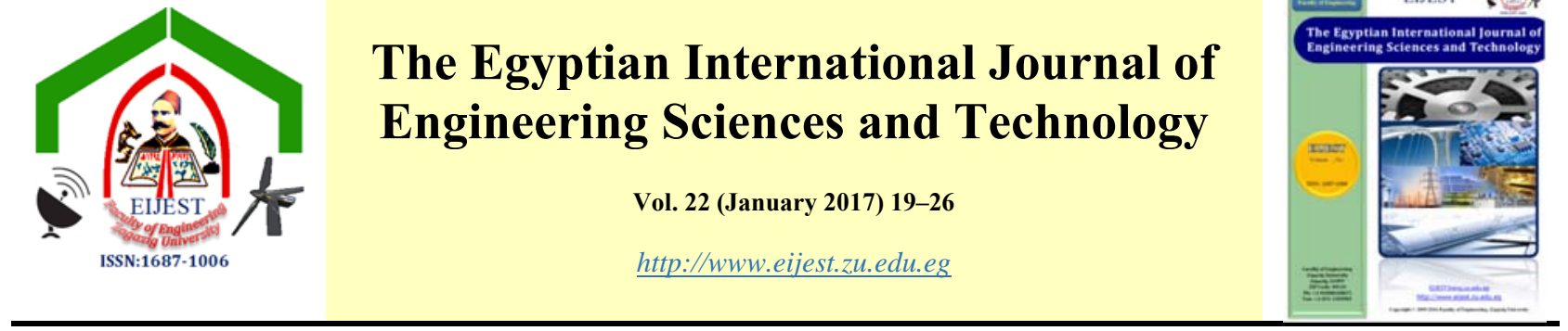

\title{
Analysis of the Nonlinear Impairments on the DWDM Optical Communication Systems
}

\author{
Walid S. El-Deeb ${ }^{a}$, M. Tosson ${ }^{b^{*}}$ and A. E. Abdelnaiem ${ }^{a}$ \\ ${ }^{a}$ Department of Electronics and Communication, Zagazig University, Zagazig, Egypt \\ ${ }^{\text {b}}$ TelecomEgypt, Zagazig, Egypt

\section{A R T I C L E I N F O} \\ Article history: \\ Received 31 July 2016 \\ Received in revised form \\ 5 September 2016 \\ Accepted 12 September \\ 2016 \\ Available online 13 \\ September 2016 \\ Keywords: \\ DWDM \\ FWM \\ Optical Amplifier \\ SRS \\ SBS \\ XPM

\begin{abstract}
A B S T R A C T
In the dense wavelength division multiplexing (DWDM) there are many factors affecting their performance. These factors can be divided into linear and nonlinear effects. The linear effects such as chromatic dispersion (CD) and polarization mode dispersion (PMD) can be compensated easily by dispersion compensation fiber (DCF) or fiber Bragg gratings (FBG). The nonlinear effects are the major and most dangerous factors that affect the signal quality and the overall performance of the DWDM. The nonlinear effects can be divided into self-phase modulation, cross phase modulation, four waves mixing, stimulated Brillion scattering and stimulated Raman scattering. This paper discusses the nonlinear effects of the DWDM system and the different ways to overcome these effects to improve the overall signal quality. This simulation analysis was performed using Optisystem (7) simulator.
\end{abstract}

(C) 2017 EIJEST. All rights reserved.

\section{Introduction}

The modern telecommunication systems rely on the DWDM technology for the transmission, especially when the number of channels and the distance increase. The linear and the nonlinear impairments affect the overall signal quality and make the signal unacceptable at the receiver.

The linear effects can be divided into two categories; attenuation losses and dispersion effects [1]. The attenuation losses are the losses of the signal power which depend on the length of fiber cable. This issue can be fixed using amplifiers. The dispersion effects can be divided into chromatic dispersion (CD) and polarization mode dispersion (PMD). The CD effects are phenomena in which different wavelengths travel at different velocities and arrive at the receiver at different times. These phenomena cause the spreading and overlapping of these wavelengths with each other. The $\mathrm{CD}$ can be compensated by negative dispersion compensation fiber (DCF) and fiber Bragg grating (FBG) [2].
The nonlinear effects are divided into two categories; parametric effects and scattering effects [3]. The parametric effects which, called also refractive index phenomena, occur when the optical pulse intensity becomes large to some extent. This increase allows the magnetic field associated with the optical pulse to modify the property of the fiber medium which in turn affects the propagation of the optical signal [4]. The change of refractive index of the fiber medium due to the increase of the pulse intensity is called "non-linear Kerr effect" [5]. This effect depends on the nonlinear part of the refractive index (n) causing the refractive index to increase with the high signal power which produces the undesired output affects such as four wave mixing (FWM), self-phase modulation (SPM) and cross-phase modulation (XPM) [6].

The scattering effects caused by the atoms and molecules that form the material, lead to optical power loss and can be categorized into two types; stimulated Raman scattering (SRS) and stimulated Brillion scattering (SBS) [7]. 


\section{Study of The Nonlinear Effects}

\section{A. The Four Waves Mixing (FWM)}

The FWM is the most effective problem in the DWDM system because it generates unwanted optical signal beside the transmitted optical signals. When the two or more optical channels, spaced with the same intervals, are transmitted through the fiber they mix with each other and produce the ghost signals. For example, if the transmitted optical signals have the frequencies $\omega_{1}$ and $\omega_{2}$, respectively, they mix with each other and generate two unwanted signals with frequencies $\left(2 \omega_{1}-\omega_{2}\right)$ and $\left(2 \omega_{2}-\omega_{1}\right)$ as shown in Fig. 1. The ghost signals can be increased according to the increase of the number of optical channels and the increase of the optical power. The number of the generated signals can be calculated according to Equ. (1) [8].

$$
k=\left(N^{3}-N^{2}\right) / 2
$$

where:

$k$ is the number of the generated signals,

$N$ is the number of the transmitted channels.

The FWM can be compensated by increasing the channels spacing and by adding an optical filter in the optical transmission link.

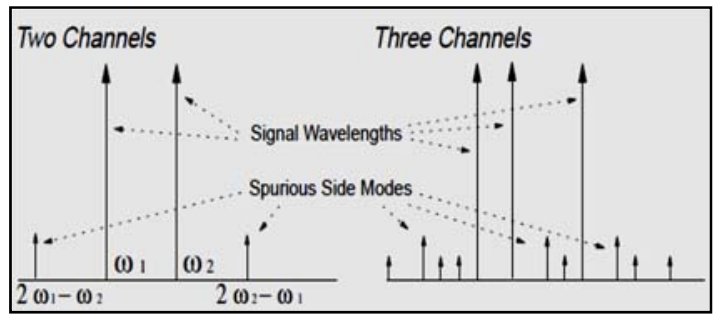

Fig. 1. The four wave mixing (FWM) effect [8]

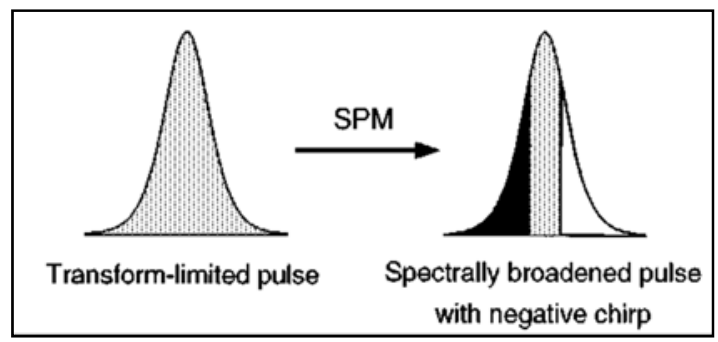

Fig. 2.The self-phase modulation (SPM) effect [9]

\section{B. The Self-Phase Modulation (SPM)}

The SPM is produced from the high signal intensities, which induce the local variable changes in the refractive index of the fiber. The refractive index increases as the optical pulse intensity increases as shown in Equ. (2). This increase leads to phase time varying in the same optical channel, which in turn makes the signal's frequency shifts towards the trailing edge and the leading edge of the signal.

$$
\mathrm{n}=\mathrm{n}_{0}+\mathrm{n}_{2} \cdot \mathrm{I}
$$

where:

$n$ is the refractive index,

$n_{0}$ is the refractive index at low intensity,

$n_{2}$ is the refractive index constant, and

$I$ is the intensity of the medium beam.

Equ. (2) can be presented by replacing the intensity $(I)$ with the optical power $(P)$ which propagates by the pulse to the mode effective area $\left(A_{e f f}\right)$ as described in Equ. (3).

$$
I=\frac{P}{A_{e f f}}
$$

Substitute the Equ. (3) into Equ. (2), the effective refractive index can be calculated using Equation (4), which indicates that the effective refractive index $\left(n_{e f f}\right)$ changes by the factor $\left(n_{2} \cdot \frac{p}{A_{e f f}}\right)$.

$$
n_{\text {eff }}=n_{0 e f f}+n_{2} \cdot \frac{p}{A_{e f f}}
$$

The change in the phase can be calculated with Equ. (5).

$$
\Delta \varphi=k_{0} \cdot \mathrm{n}_{2} \cdot \frac{P}{A e f f} \cdot l_{e f f}
$$

where:

$k_{0}$ Is the equals to $2 \pi / \lambda 0$,

$n_{\text {eff }}$ Is the refractive index with the existence of the nonlinearity,

$n_{0 e f f}$ Is the refractive index at low power (can be neglected),

$\Delta \varphi$ Is the Phase change,

$\lambda_{0}$ Is the wavelength at the free space,

$\alpha$ Is the attenuation coefficient,

$l$ Is the actual length of the fiber, and $l_{e f f}$ is the effective length.

The effective length $\left(l_{e f f}\right)$ can be calculated using Equation (6), where $\alpha$ is the attenuation coefficient.

$$
L_{e f f}= \begin{cases}1 / \propto & l \gg 1 \backslash \propto \\ l & l \ll 1 \backslash \propto\end{cases}
$$

The SPM is the exact opposite of the positive chromatic dispersion and it appears in one channel only as shown in Fig. 2. This is why the SPM can be compensated by the allowance of some chromatic dispersion during the propagation of the optical signal [9].

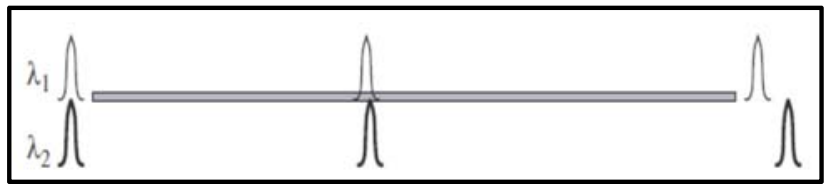

Fig. 3. The cross-phase modulation (XPM) Effect [10] 


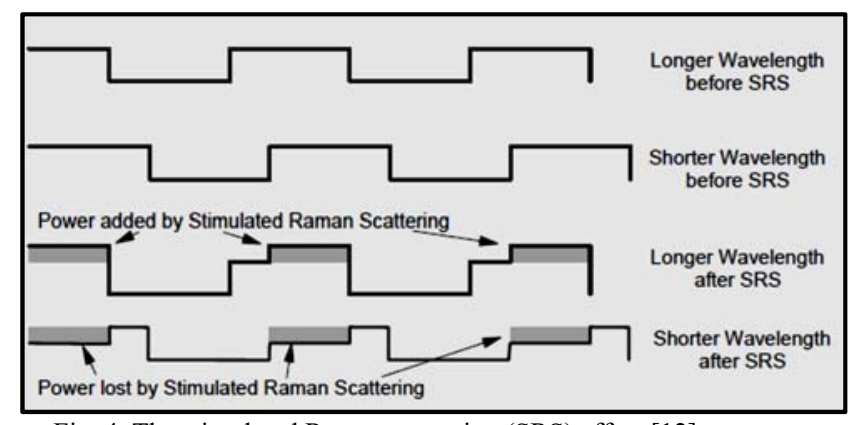

Fig. 4. The stimulated Raman scattering (SRS) effect [12]

\section{A. The Cross-Phase Modulation (XPM)}

The XPM occurs on the multiple channels of the DWDM system where many different wavelengths are propagating at the same time. Every wavelength leads to the change in the refractive index of the fiber link depends on the optical power carried by this wavelength. The change in the refractive index that occurs by the propagating wavelength will affect the other propagating wavelengths, producing what is called XPM. The XPM causes the travelling wavelengths to overlap with each other which increase the bit error rate. The XPM can be compensated by the allowance for some chromatic dispersion or by increasing the channels spacing as shown in Fig. 3 [10].

\section{B. The Stimulated Brillouin Scattering (SBS)}

The SBS appears when the power of the optical signal is less than milli watts. The optical signal is a strong electromagnetic field which leads to the regular vibration of the refractive index as a virtual grating. The Brillouin scattering effect is occurred by the light being reflected by the grating, created by the regular pattern of the refractive index. This effect causes the light to be reflected and scattered towards the transmitter, which resulting in signal power loss. The SBS depends on many factors such as; signal line width, signal power, wavelength and fiber core size. The SBS can be reduced by using fiber with large core size and high active gain to reduce the value of the effective length of the fiber [11].

\section{The Stimulated Raman Scattering (SRS)}

The SRS effect is generated by a mechanism similar to that producing the SBS effect. The interactions involved are due to the molecular vibrations resulting in the light to be scattered in both forward and backward directions. The SRS effect is not a big problem in the single channel but it becomes a significant problem in the DWDM system. This is because the optical power is transferred from the shorter wavelengths to the longer wavelengths. This optical power transfer results in adding the noise to the longer wavelengths and subtract it from the shorter wavelengths as shown in Fig. 4.

The SRS can be reduced by keeping the signal power level below the threshold power level $\left(p_{t h}\right)$ which requires the reduction of the distance between amplifiers [12].

The Stimulated Raman Scattering (SRS) process is initiated by noise, thermally induced fluctuations in the optical fields and Raman active vibration modes. An incident pump field $(\omega \mathrm{P})$ interacts with the vibration fluctuations, losing a photon which is down shifted in frequency by the vibration frequency $(\Omega)$ to produce a Stokes wave $(\omega \mathrm{S}$,$) and also an optical$ phonon (quantum of vibration energy). These stimulate further break-up of pump photons in the classical exponential population dynamics process in which "the more you have, the more you get".

The pump decays with propagation distance and both the phonon population and Stokes wave grow together. If the generation rate of Stokes light exceeds the loss, stimulated emission occurs and the Stokes beam grows exponentially

The threshold power level $\left(\mathrm{p}_{\mathrm{th}}\right)$ can be calculated as a function of the attenuation factor $(\alpha)$, effective length $\left(L_{e f f}\right)$ and the Raman gain $(G)$ as described in Equ. (7) and (8).

$p_{t h}=\frac{16 \cdot A_{e f f}}{G \cdot L_{e f f}}$

$p_{t h}=\frac{16 \cdot A_{\text {eff }}}{G} \times \alpha$

The block diagram for the DWDM system is illustrated in Fig. 5 and the simulation circuits are shown in Fig. 6 and 7.

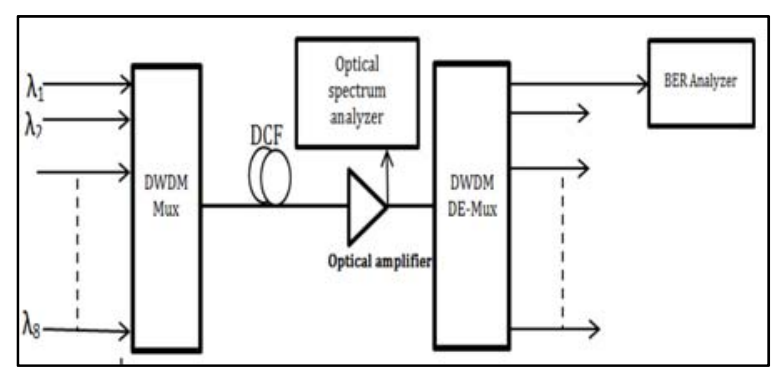

Fig. 5.The Block diagram of the DWDM system

\section{Description of The Nonlinear Simulation Setup}

The transmitter consists of DWDM transmitter with 8 channels at different wavelengths starting from $1552.52 \mathrm{~nm}$ to $1546.91 \mathrm{~nm}$, with $0.8 \mathrm{~nm}$ of channels spacing and optical channel power of $0 \mathrm{dBm}$. Channel bit rate is $10 \mathrm{Gbps}$, with line modulation type nonreturn to zero (NRZ) as described in Table 1. The transmission medium length is $100 \mathrm{~km}$ with attenuation of $0.25 \mathrm{db} / \mathrm{km}$ and the dispersion value is $16 \mathrm{ps} / \mathrm{nm} / \mathrm{km}$. 


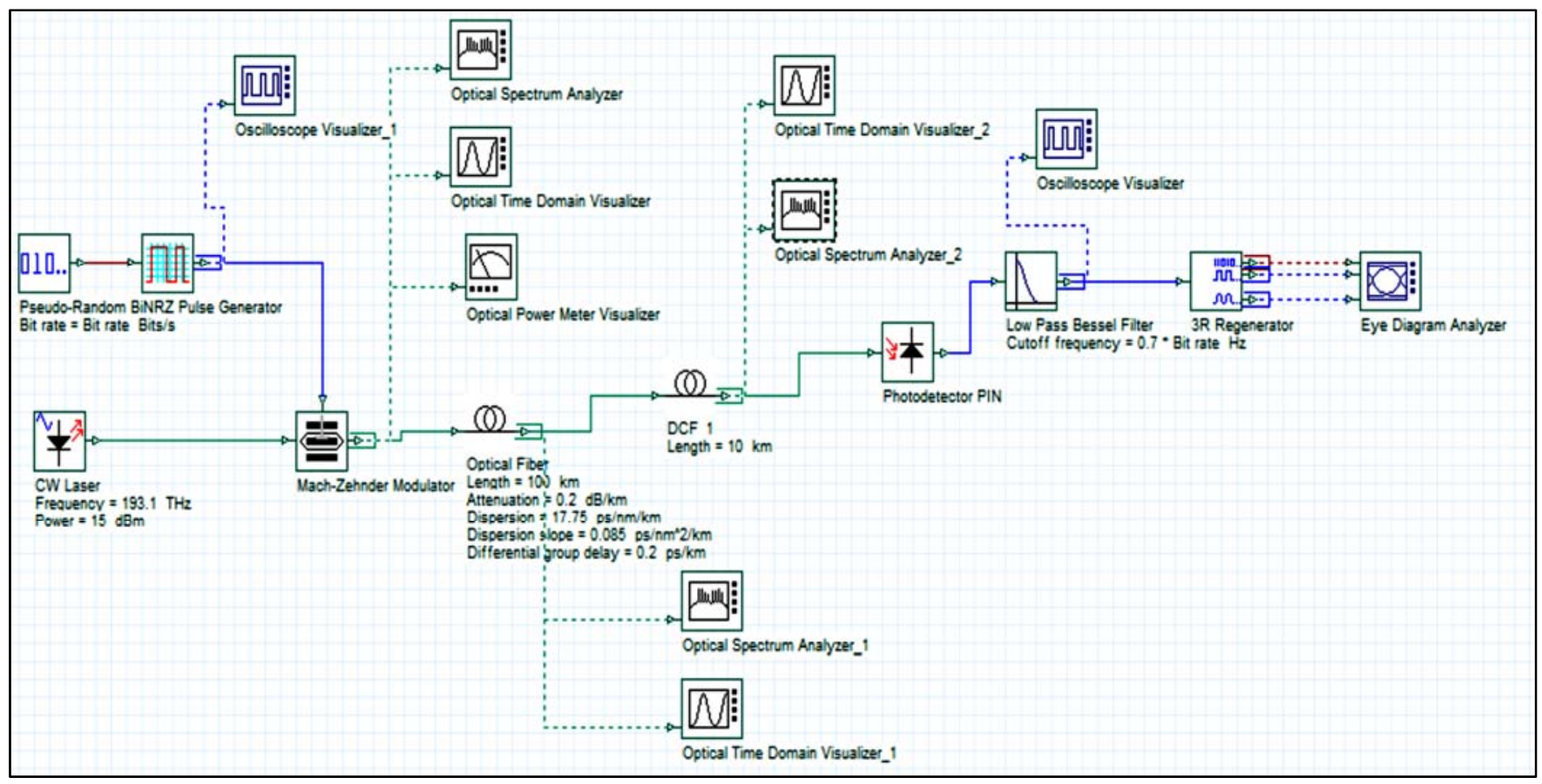

Fig. 6. The simulation circuit for analyzing the SPM effect

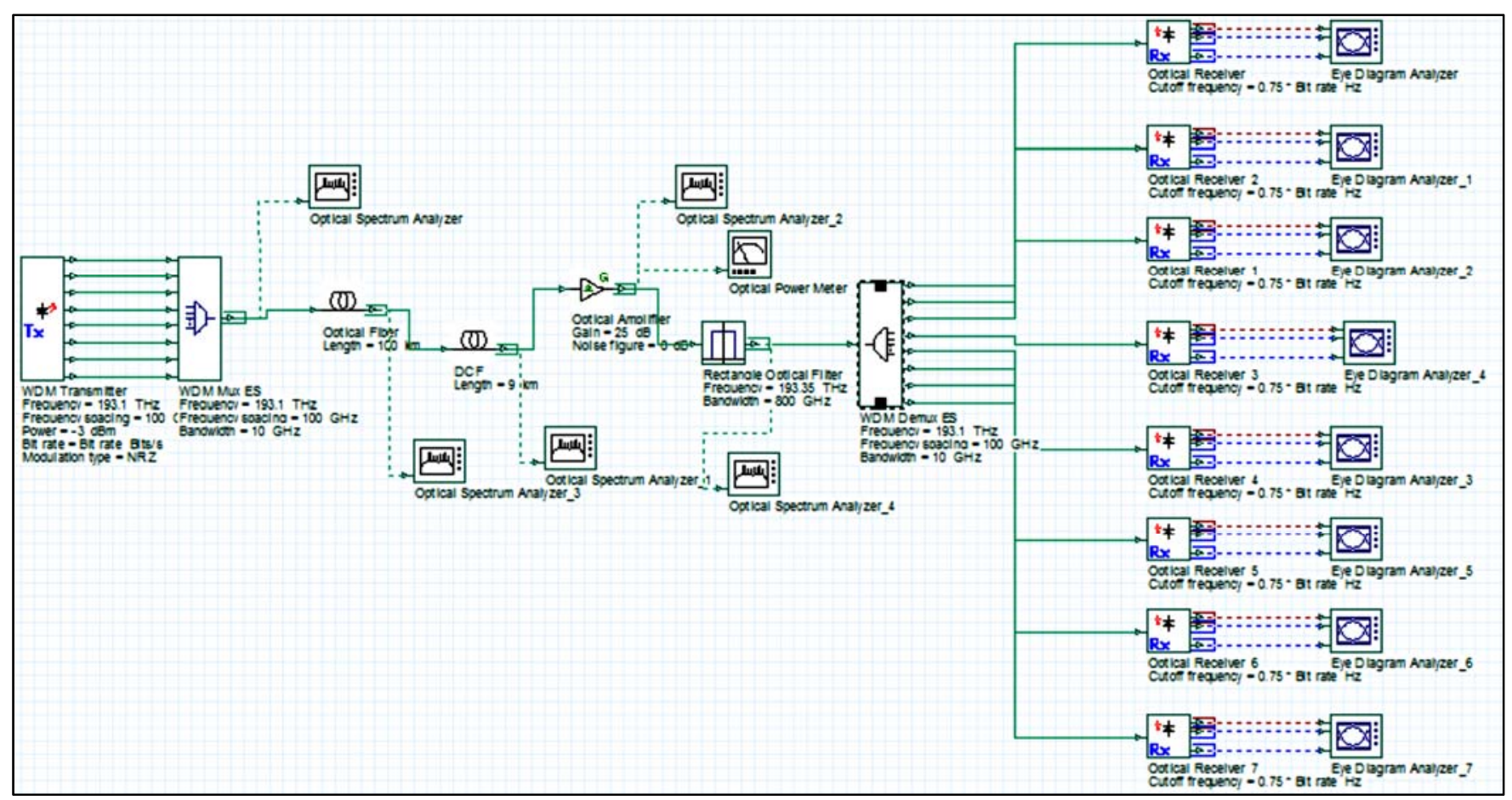

Fig. 7. The 8-Channels simulation circuit for analyzing the XPM and the FWM effects 
The receiver side for the DCF compensation technique contains the DCF with length of $10 \mathrm{Km}$ with dispersion value of $160 \mathrm{ps} / \mathrm{nm} / \mathrm{km}$ and attenuation of $0.6 \mathrm{db}$. It also includes an optical amplifier of $25 \mathrm{~dB}$ gain and 0dBnoise figure. The other receiver components are; rectangular optical filter, BER analyze for XPM and optical spectrum analyze after the optical amplifier for FWM as shown in Fig. 6 and 7. Tables 1 and 2 summarize the simulation parameters for the transmitter and the receiver of the WDM system.

TABLE 1: SimUlation PARAMETERS OF THE WDM TRANSMitTER

\begin{tabular}{l|ll}
\hline \multicolumn{1}{c|}{ Parameters } & WDM Transmitter & WDM Mux. \\
\hline No. of channel & $8 \mathrm{CH}$ and $15 \mathrm{CH}$ & $8 \mathrm{CH}$ and $15 \mathrm{CH}$ \\
Optical power & $0 \mathrm{dBm}$ and $15 \mathrm{dBm}$ & - \\
Frequency spacing & $100 \mathrm{GHz}$ & $100 \mathrm{GHz}$ \\
Bit rate & $10 \mathrm{Gbps}$ & - \\
Bandwidth & - & $10 \mathrm{GHz}$ \\
\hline
\end{tabular}

TABle 2: SimUlation PARAMETERS OF THE WDM ReCEIVER

\begin{tabular}{c|ccc}
\hline Parameters & DCF & $\begin{array}{c}\text { Optical } \\
\text { amplifier }\end{array}$ & $\begin{array}{c}\text { Rectangular } \\
\text { optical filter }\end{array}$ \\
\hline Length & $10 \mathrm{Km}$ & - & - \\
Dispersion & $160 \mathrm{ps} / \mathrm{nm} / \mathrm{Km}$ & - & - \\
Gain & - & $25 \mathrm{dBm}$ & - \\
Noise figure & - & $0 \mathrm{~dB}$ & - \\
Center & - & - & $193.35 \mathrm{THz}$ \\
frequency & - & - & $800 \mathrm{GHz}$ \\
Bandwidth & - & - & \\
\hline
\end{tabular}

\section{Discussion of Simulation Results}

\section{A. The Results of the Self-Phase Modulation}

Without activating the SPM in the simulator, the signal propagates with high signal quality and no bit error rate as illustrated in Fig. 8(a). By activating the SPM, the signal quality decreases and the bit error rate increases as in shown in Fig. 8(b). The results for the two cases are summarized in Table 3.

TABle 3: THE Results FoR the SELF-PHASE ModulationEFFECT

\begin{tabular}{l|lll}
\hline Case & BER & Q-factor & Eye height \\
\hline Without SPM effect & $3.34092 \mathrm{e}^{-14}$ & 7.4938 & $4.7857 \mathrm{e}^{-6}$ \\
With SPM effect & $2.32554 \mathrm{e}^{-11}$ & 6.58059 & $4.08659 \mathrm{e}^{-6}$ \\
After SPM Compensation & $2.64053 \mathrm{e}^{-17}$ & 8.37998 & $5.84224 \mathrm{e}^{-6}$ \\
\hline
\end{tabular}

- Compensation for the Self-Phase Modulation

In order to compensate for the SPM, some chromatic dispersion should be neglected in the transmission link in order to improve the BER and the signal quality as shown in Table3 and Fig. 9

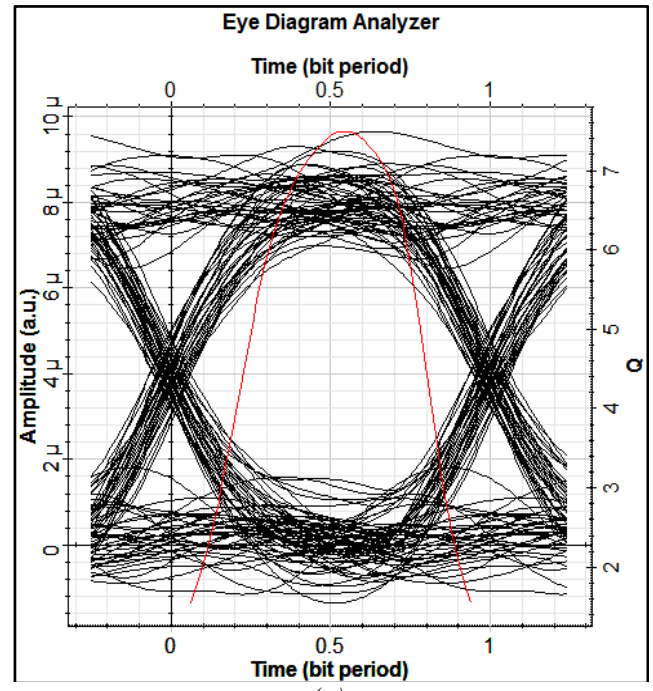

(a)

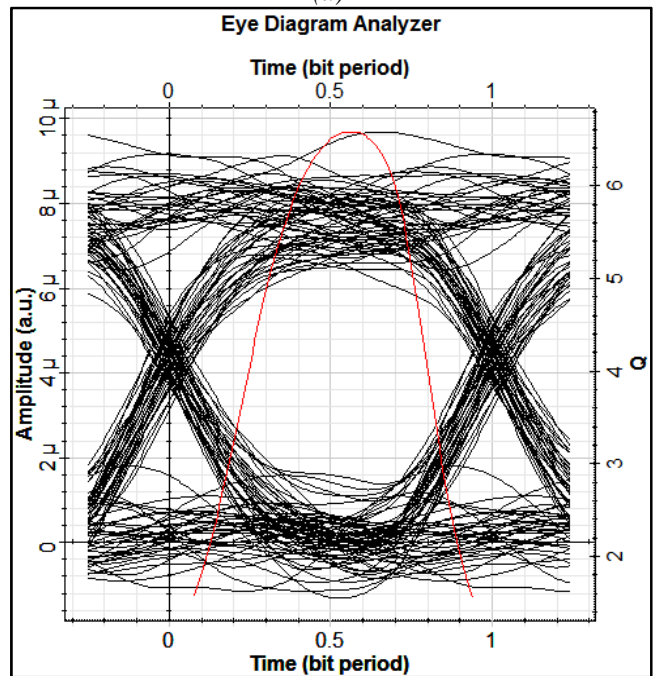

(b)

Fig. 8: (a) Eye diagram without SPM effect, (b) Eye Diagram with SPM effect

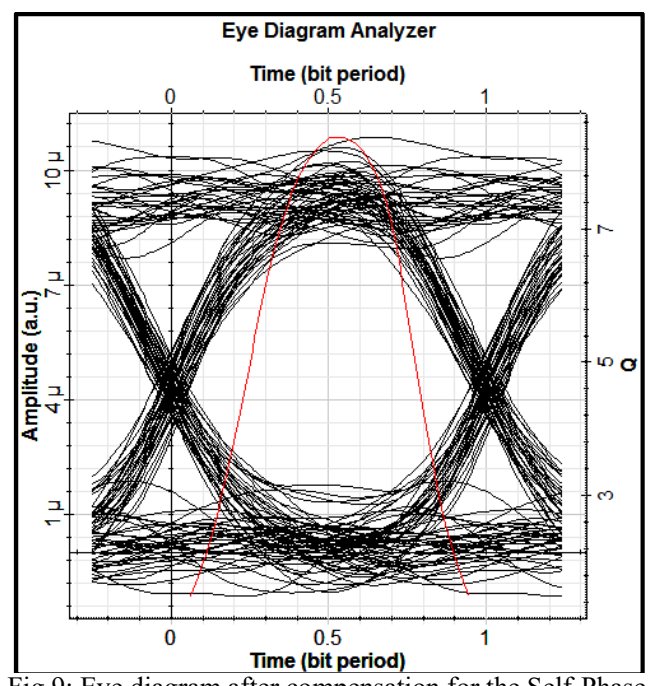

Fig.9: Eye diagram after compensation for the Self-Phase Modulation 


\section{B. The Results of the Cross-Phase Modulation}

The BER increases and the signal quality decreases with the increase of the channels number and the optical power. The results shown in Table 4 and Fig. 10 describe the signal parameters with XPM effect for 8 and 15 channels with different power levels.

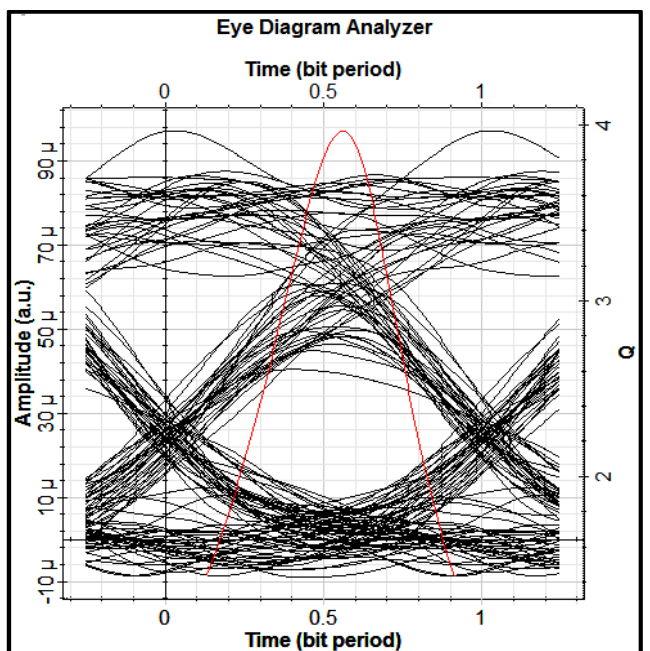

(a)

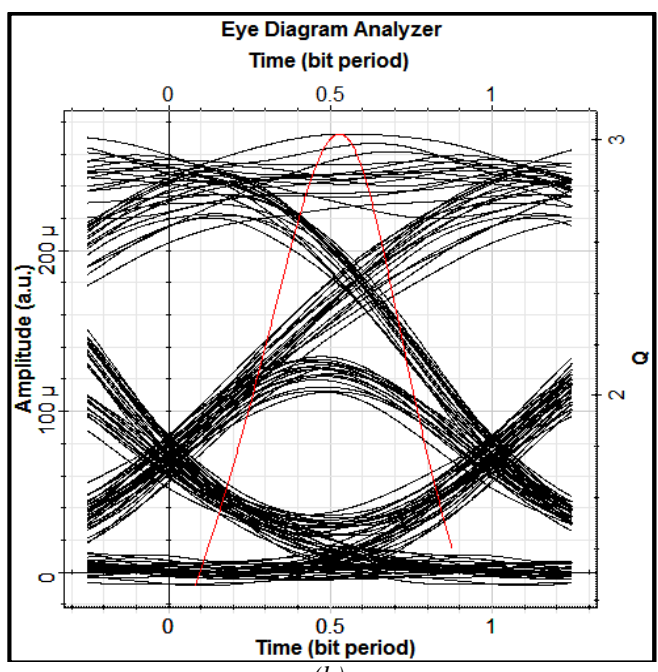

(b)

Fig.(10): (a) The effect of XPM for 8-channels with 0dBm power level (b) The effect of XPM for 15-channels with $5 \mathrm{dBm}$ power level.

TABLE 4: THE PARAMETERS OF THE SIGNAL WITH THEXPM EFFECT

\begin{tabular}{c|cc}
\hline Parameters & 8-Channels & 15-Channels \\
\hline Q-factor & 3.96822 & 3.02199 \\
BER & $3.1905 \mathrm{e}^{-5}$ & 0.00102911 \\
Eye height & $1.51523 \mathrm{e}^{-5}$ & $1.234 \mathrm{e}^{-6}$ \\
\hline
\end{tabular}

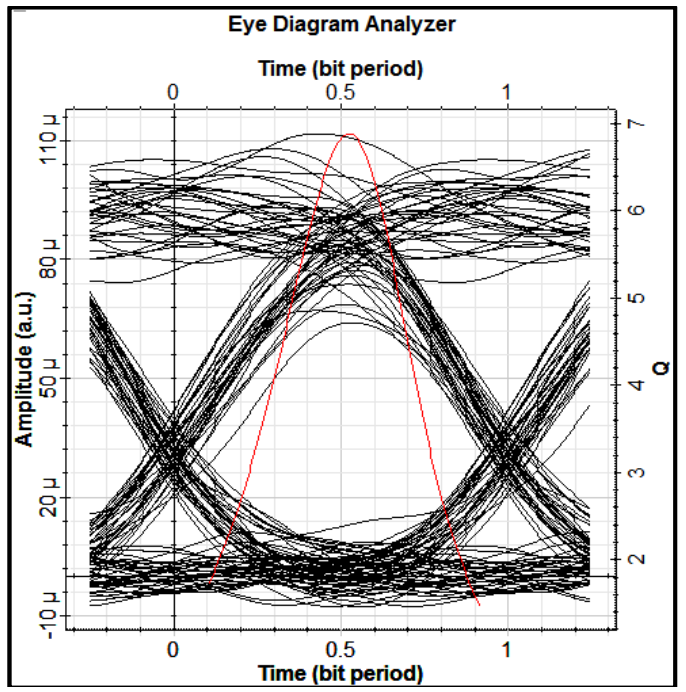

(a)

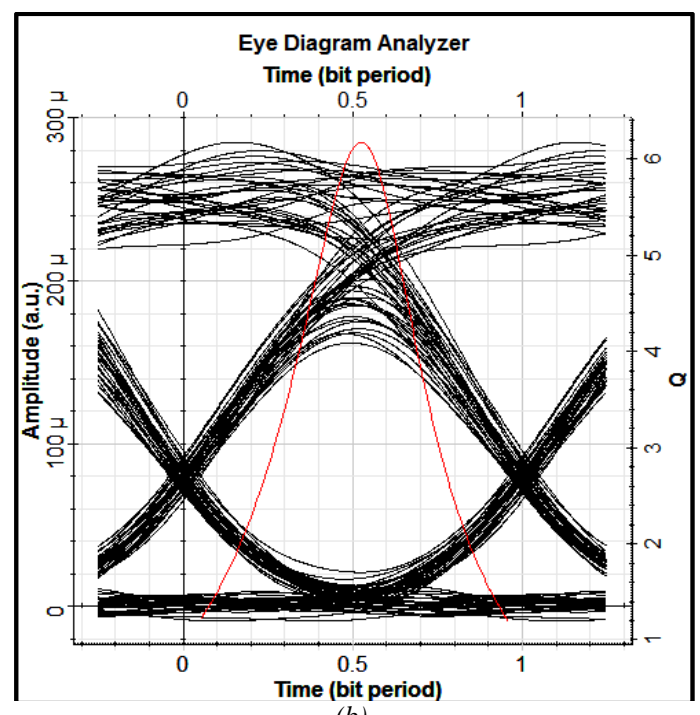

(b)

Fig.(11): (a) Eye diagramfor 8-channels with power 0dBm Power level after compensation for XPM (b) Eye diagramfor 15-channels with $5 \mathrm{dBm}$ power level after compensation for XPM

\section{- Compensation for the Cross-Phase Modulation}

In order to compensate for the XPM, the channel spacing should be increased to $130 \mathrm{GHz}$ for the 8channels system and $150 \mathrm{GHz}$ for the15-channels system. Also, some chromatic dispersion should be allowed in fiber link to overcome the crosstalk in the optical signal. The results are summarized in Table 5 and Fig.11 for the 8-channels and 15-channels with 0 and $5 \mathrm{dBm}$ power levels, respectively.

TABLE 5: THE PARAMETERS OF THE SIGNAL AFTER COMPENSATION FOR XPM

\begin{tabular}{c|cc}
\hline Parameters & 8-Channels with $0 \mathrm{dBm}$ & 15-Channels with $5 \mathrm{dBm}$ \\
\hline Q-factor & 6.75633 & 6.17412 \\
BER & $6.59404 \mathrm{e}^{-12}$ & $2.5777 \mathrm{e}^{-10}$ \\
Eye height & $4.85778 \mathrm{e}^{-5}$ & 0.000109386 \\
\hline
\end{tabular}




\section{The Results of the Four Wave MixingEffect}

Figure 12 (a) and (b) show the spectrum of the optical signal at the output of the transmitter and after propagating over the optical link, respectively. Fig. 12 (b) shows that, there are many unwanted pulses appeared on the signal's spectrum after propagating over the optical link.

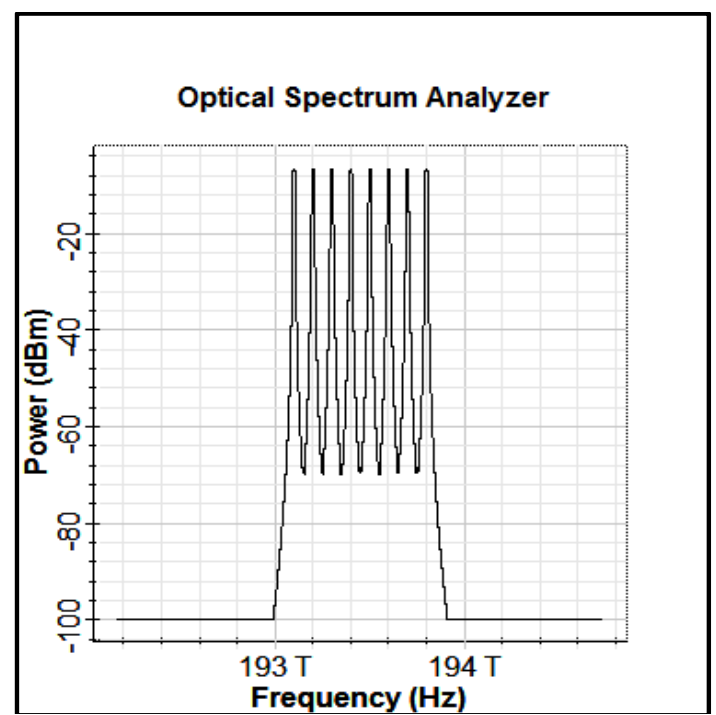

(a)

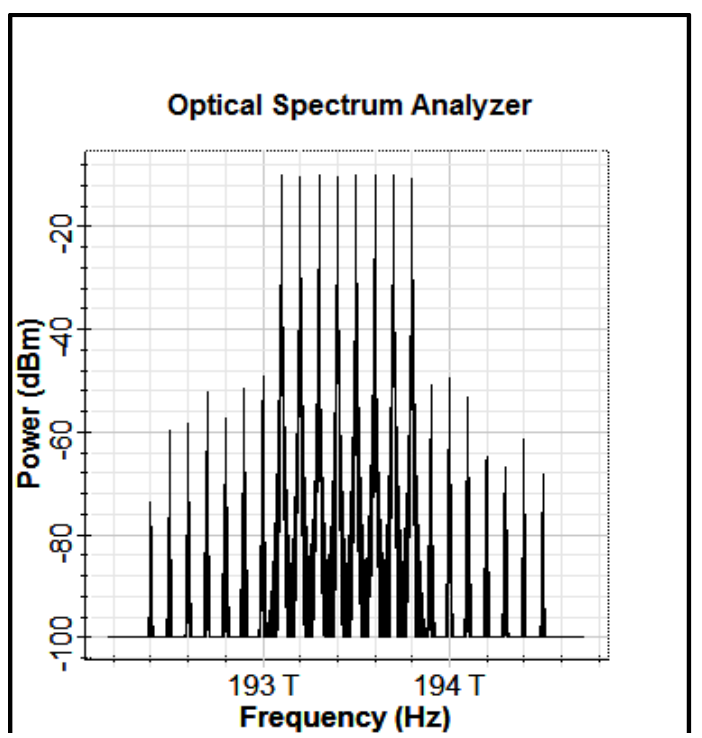

(b)

Fig.(12): (a) spectrum signal output at WDM transmitter and (b) spectrum signal after propagating at optical amplifier.

\section{- The Compensation for the Four Wave Mixing Effect}

The optical signal can be cleared from the undesired FWM effect by using rectangular optical filter after the optical amplifier to remove any unwanted pulses as described in Fig. 13.

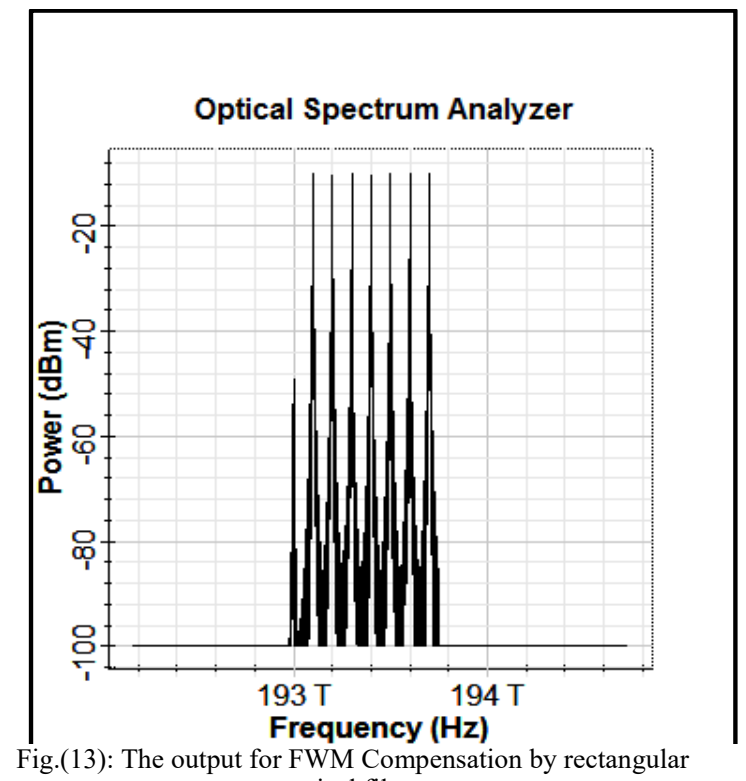
optical filter.

\section{Conclusion}

This paper describes the nonlinear effects in the DWDM systems such as SPM, XPM and FWM. The best technique to compensate for the SPM effect is to insert or allow for some chromatic dispersion over the optical link. The FWM and XPM appear on DWDM communication system and become significant with the increasing in the number of channels and the power of the optical signal. These nonlinear phenomena have an enormous bad effect on the signal quality and BER. These effects can be eliminated by increasing the channel's spacing and adding a rectangular optical filter after the optical amplifier in the design of the DWDM optical system.

\section{References}

[1] M.Tosson, Walid S. El-Deeb, A.E.Abdelnaiem,"Comparison of Chromatic Dispersion Compensation in Optical Fiber with Fiber Bragg Grating (FBG) and Dispersion Compensation Fiber (DCF) for $10 \mathrm{Gbps}$ and 40 Gbps", International Journal of Scientific and Engineering Research (IJSER), Vol 6, Issue 6, pp. 1284-1289, June2015.

[2] M.Tosson, Walid S. El-Deeb, A.E. Abdelnaiem, "Dispersion Compensation Techniques for DWDM Optical Networks", International Journal of Advanced Research in Computer and Communication Engineering (IJARCCE),Vol. 4, Issue 8, pp. 1-6, August 2015.

[3] Manjari Sharma and P. K. Raghav, "Analysis on Dispersion Compensation in WDM Optical Network using Pre, Post and Symmetrical DCF based on Optisystem", MIT International Journal of Electronics and Communication Engineering (IJECE), Vol. 4, Issue 1, pp. 58-63, January 2014.

[4] Ravi Prakash Shukla, Mukesh kumar, A.K. Jaiswal, Rohini Saxena,"Performance Analysis of Dispersion in Optical Communication link Using Different Dispersion Compensation Fiber (DCF) Models", International Journal of Recent Technology an d Engineering (IJRTE),

Vol 1, Issue 2, pp. 161-162, June 2012. 
[5] Ronen Dar, Meir Feder, Antonio Mecozzi, and Mark Shtaif, "Accumulation of nonlinear interference noise in fiber-optic systems", Optical Society of America journal, Vol. 22, Issue. 12, pp.14199-14211, 2014.

[6] Marvin Suraj Koshy, Pratheesh P,"Analysis of SPM and FWM in Optical Fiber Communication System using Optisystem", International Journal of Engineering Research \& Technology (IJERT),Vol. 3, Issue 3, pp.1700-1703, March 2014.

[7] Devan Bhalla, Monica Bhutani, "Performance Analysis of Four Wave Mixing:A Non -Linear Effect in Optical Fibers", International Journal of Computer Applications”, Vol. 93, Issue 7, pp. 44-50, May 2014.

[8] Yogita Thareja, Gurmeet Singh, "Effect of SRS-XPM-FWMASE Nonlinearity in Optical Communication System", International Journal of Advanced Research in Electrical, Electronics and Instrumentation Engineering, Vol. 2, Issue 7, pp. 3057-3062, July 2013.
[9] Arash Bahrami, Wai Pang Ng, Zabih Ghassemlooy and Thavamaran Kanesan, "Effect of Nonlinear Phase Variation in Optical Millimetre Wave Radio over Fibre Systems", Journal of Electrical and Computer Engineering Innovations(JECEI), Vol. 3, Issue. 1, pp. 17-27, 2015.

[10] N. Kikuchi, K. Sekine and S. Sasaki,“Analysis of cross-phase modulation (XPM) effect on WDM transmission performance"Electronics Leters,Vol. 33 No. 8, April 1997.

[11] Liang B. Du and Arthur J. Lowery, "Channelized Chromatic Dispersion Compensation for XPM Suppression and Simplified Digital SPM Compensation", Optical Society of America, conference, San Francisco, California United States, 9-13 March 2014, pp.2-13.

[12] Rahul Umesh Kale, Pavan Mahadeo Ingale, Rameshwar Tukaram Murade, "Comparison of SRS \& SBS (Non Linear Scattering) In Optical Fiber", International Journal of Recent Technology and Engineering (IJRTE), Vol 2, Issue 1, pp.118-122, March 2013. 